LICENÇA CC BY:

Artigo distribuído sob os termos

Creative Commons, permite uso e distribuição irrestrita em qualquer meio desde que o autor credite a fonte original.

\section{AVANCE PRIVATIZADOR EN LA EDUCACIÓN: EL CASO DE URUGUAY}

AVANCE PRIVATIZADOR EN LA EDUCACIÓN: EL CASO DE URUGUAY ADVANCES IN PRIVATIZATION IN EDUCATION: THE CASE OF URUGUAY

Martín Sanguinetti Pardo ${ }^{2}$

${ }^{1}$ Mestre em História Econômica pela UDELAR. Assessor do Sindicato da Educação Pública do Uruguai, Montevideo, Uruguai.

${ }^{2}$ Mestre em Gestão Costeira Integrada pela UDELAR. Docente da Faculdade de Ciências Econômicas e de Administração da Universidad de La República Uruguay (UDELAR), Montevideo, Uruguai.

\begin{abstract}
Resumen: La privatización educativa es un fenómeno global que ha ido convirtiéndose en una preocupación cada vez más relevante, tanto en lo político como lo académico. El presente documento analiza dos tipos de herramientas novedosas en el Uruguay (Participación PúblicoPrivada y los centros privados gratuitos) que logran dar un paso relevante por lo cuantitativo y lo simbólico, en la privatización exógena del modelo educativo uruguayo. Estos dos mecanismos de privatización tienen un importante impacto en la apropiación del sector privado sobre los recursos públicos, y a su vez, avanza de manera sustantiva sobre la oferta educativa, sobre la educación pública y sobre los recursos públicos.
\end{abstract}

Palabras clave: Educación; Privatización; PPP.

Resumo: A privatização educacional é um fenômeno global que se tornou uma preocupação cada vez mais relevante, tanto política quanto academicamente. $\mathrm{O}$ documento analisa dois tipos de novas ferramentas no Uruguai (participação público-privada e centros privados livres) que conseguem dar um passo à frente na privatização exógena do modelo educacional uruguaio. Esses dois mecanismos de privatização têm um impacto importante na apropriação do setor privado dos recursos públicos e, por sua vez, avançam substancialmente na oferta educacional, na educação pública e nos recursos públicos.

Palavras-chave: Educação; Privatização; PPP.

Abstract: Privatization in the education system is a global phenomenon that has become an increasing concern, from the political and academic points of view. This document analyzes two new types of policy in Uruguay - Public-Private Partnerships and charter schools that have been able to take a step further in the exogenous privatization of the Uruguayan educational model. These two mechanisms of privatization have had an important impact on the appropriation of public resources by the private sector, which in turn, has led to important advances in the educational offer, public education, and public resources.

Keywords: Education; Privatization; PPP. 


\section{Introducción}

La privatización educativa es un fenómeno global que ha ido convirtiéndose en una preocupación cada vez más relevante, tanto en lo político como lo académico. América Latina tiene los niveles más altos de participación del sector privado en la matrícula educativa y además muestra en los últimos 30 años las tasas de crecimiento más aceleradas. En ese contexto, Uruguay se presenta como un país que tiene una tasa de cobertura privada menor que el promedio latinoamericano, pero muy alta si se compara con el resto del mundo (Bellei y Orellana, 2014).

En nuestro país, son escasas las reflexiones sobre el fenómeno de la privatización educativa, aunque podemos destacar algunos trabajos recientes que han hecho importantes contribuciones. En primer lugar, está el aporte relativamente pionero de Gustavo Cosse (2001) donde analiza los fundamentos conceptuales del sistema de vouchers ${ }^{1}$ y de otras formas de "subsidio a la demanda", concluyendo que no hay desde el punto de vista conceptual ni empírico, una justificación clara a su implementación en nuestro país. Más acá en el tiempo, Bordoli y Conde (2016) muestran que desde una génesis modernizadora donde el valor de lo público era central, asistimos a un creciente impulso privatizador con el advenimiento de las modalidades de liceos públicos de gestión privada. En segundo lugar, Verger et al (2017) realizan un análisis comparativo para toda América Latina sobre la evolución de la educación privada y sus fundamentos y obtienen como resultado una tipología, donde caracterizan a Uruguay como un país de "privatización latente". Por último, Bordoli et al (2017) realizan un detallado análisis sobre la normativa detrás de los impulsos privatizadores, así como los distintos actores sociales que disputan los sentidos de la educación, tanto en pro de la privatización educativa como quiénes lo hacen en defensa de lo público.

En nuestro país, las tasas de matrícula privada son elevadas en todos los niveles educativos ${ }^{2}$. A su vez, la privatización tiene un gran componente de gasto privado, es decir, es mayoritariamente pagado por parte de los hogares. Esto posiciona a Uruguay entre los países de mayor gasto privado del continente con un 1,6\% del Producto Bruto Interno (PBI) para el 2012, (INEED; 2016). Así, a lo largo de la historia el sector privado se ha expandido gracias al pago de los hogares, a la vez que ha logrado disputar recursos estatales vía exoneraciones fiscales. De hecho, en la Constitución de 1942 y como consecuencia del accionar organizado de la primera cámara empresarial de educación privada asociada a la iglesia católica $\left(\mathrm{AUDEC}^{3}\right)$, se incorpora un artículo que exonera de todo tipo impuestos a las instituciones de enseñanza privada. Esto hace que el Estado a través de exoneraciones fiscales, subvenciona tanto a la oferta -por el no pago de impuestos y contribuciones- como a la demanda -por el no pago del impuesto al consumo-. Este privilegio sigue vigente hasta nuestros días y significa aproximadamente un $0,39 \%$ del PBI (INEED; 2014). 
En el presente trabajo, siguiendo la clasificación de Verger et al (2015) sobre las formas de privatización, se pone el foco en dos mecanismos de privatización exógena de reciente aparición en nuestro país. Por un lado, se analizan los centros educativos que se van a construir mediante Contratos de Participación Público Privado. Por otro lado, se analizan las donaciones especiales en el sistema educativo uruguayo. Estos dos mecanismos de privatización tienen un importante impacto en la apropiación del sector privado sobre los recursos públicos y a su vez, avanzan de manera sustantiva sobre la oferta educativa, por tanto, sobre la educación pública.

La discusión sobre la privatización está muy ligada a la cuestión sobre cuáles son las condiciones que permiten la realización plena del Derecho Humano a la Educación. La privatización de la educación está indudablemente enraizada a una elitización del acceso al acervo cultural y cognitivo de la humanidad, segmentando dicho acervo según la capacidad de pago y la riqueza relativa de hogares, regiones o países. Asistimos a una época donde el capital ha colonizado las distintas esferas de la vida, y en ese contexto, la privatización de la educación nos inhibe a la hora de configurar y proponer horizontes igualitarios y más democráticos (CLADE, 2012). Por tanto, este estudio es un aporte crítico en defensa de lo público y contra las diversas formas de mercantilización que azotan a los bienes comunes.

\section{Las inversiones vía Participación Público Privada Origen de las Inversiones vía Participación Público Privada}

Las inversiones o contratos de Participación Público Privada (en adelante PPP) son aquellos en que el Estado le encarga a un privado, por un período de tiempo determinado, el diseño, la construcción y el mantenimiento (o alguna de dichas prestaciones), además de la financiación, de determinada infraestructura pública como por ejemplos: carreteras, cárceles, hospitales o escuelas.

El origen de esta herramienta se encuentra en el Reino Unido en la década de 1980, bajo el gobierno de Margaret Thatcher, cuando tuvo lugar una fuerte oleada privatizadora. La privatización de los servicios públicos como la salud y la educación tenía fuertes resistencias en Inglaterra en los 1980 y eran poco atractivo para el capital con la privatización pura y dura. Con estas herramientas se encontró una forma de avance del capital sobre los servicios públicos, captando recursos del Estado. En la actualidad, esta herramienta es prioridad en las agendas de organismos internacionales -como el Banco Mundial, la OCDE y el FMI- que exigen a los países miembros que la impulsen, configurándose así una nueva forma del avance privatizador (Hall, 2015) y (Internacional de la Educación, 2009).

Por otro lado, desde estas primeras oleadas privatizadoras de la década de 1980, en diferentes países hubo introducción de reglas fiscales que limitan el Déficit Fiscal y la inversión pública tradicional ${ }^{4}$. Sin embargo, varios gobiernos tenían necesidad de invertir 
en infraestructura pública, pero se veían limitados por esta regla fiscal, que generó una restricción y una inversión menor a la deseada. Así es que surgen alternativas para poder eludir la restricción contable y efectivamente ejecutar la inversión. Dentro de estas alternativas aparecen: fomentar la inversión privada, otorgando marcos jurídicos de promoción (por ejemplo, mediante la exoneración de impuestos); realizar Contratos de PPP, o impulsar la inversión pública a través de herramientas especiales como fideicomisos o leasings.

Por lo tanto, los Contratos de PPP se convirtieron en una buena solución. Si bien el gobierno se compromete a pagar la inversión (como si se tratase de un préstamo que hubiese pedido), las normas contables permiten tratar a estos contratos como un préstamo privado y no como deuda pública, por lo que no repercute en el Déficit Fiscal. Sin embargo, este tipo de inversiones representa otra forma de privatización, permitiendo a las empresas privadas beneficiarse del dinero público, y exigirles a los servicios públicos que proporcionen oportunidades comerciales rentables (Fine y Hall; 2012).

Mirando el caso de Uruguay se puede comprender el funcionamiento de esta herramienta. Los Contratos de PPP permiten al Estado cumplir más rápidamente con el objetivo de invertir en infraestructura pública sin incrementar el Déficit Fiscal. Cuando se realiza un Contrato de PPP, el Estado no realiza ningún pago hasta que el servicio esté en funcionamiento y comience a pagar un canon (Pago Por Disponibilidad), burlando el "criterio caja" de contabilizar la inversión ${ }^{5}$.

Sin embargo, la decisión de realizar inversiones mediante Contrato de PPP no es inocua. Implica, por la vía de los hechos, la pérdida parcial o total de la propiedad del Estado de activos fundamentales para la sociedad junto con una menor capacidad de control de los gastos del Estado (Esponda y Molinari, 2017).

Por otro lado, para las empresas privadas este tipo de contratos representan un negocio muy atractivo ya que un solo contrato les proporciona una fuente de ingresos altamente segura (por tratarse de un contrato con un Estado) ya que los contratos pueden durar hasta 35 años.

En suma, los Contratos de PPP surgen de una combinación de una nueva oleada privatizadora junto con una forma de equilibrar los presupuestos nacionales, mediante el ocultamiento de deuda pública.

\section{Sobre la Normativa en Uruguay}

En Uruguay, la Ley sobre las inversiones de Participación Público-Privada (PPP) se aprobó en 2011 (Ley 18.786).

Según la normativa, los Contratos de PPP son aquellos donde la Administración Pública le encarga a un privado, por cierto período, el diseño, la financiación, la construcción y la operación de cierta infraestructura. Además, el plazo mínimo es de 20 años y el máximo 
de duración de los contratos y de sus prórrogas, no podrá exceder 35 años. Respecto a los ingresos que obtiene el privado, se prevén diferentes modalidades: pago del usuario por uso del servicio, pago de un canon por parte del Estado o una combinación de ambas.

Además, la normativa establece de manera taxativa las obras de infraestructura social que se pueden realizar con esta herramienta: "cárceles, centros de salud, centros de educación, viviendas de interés social, complejos deportivos y obras de mejoramiento, equipamiento y desarrollo urbano y obras hidráulicas para riego". Por otro lado, "en ningún caso, los Contratos de Participación Público-Privada podrán incluir: servicios educativos cuando se trate de centros educativos." Sin embargo, ni la Ley ni los decretos reglamentarios definen qué se entiende por servicios educativos.

\section{Las Inversiones vía PPP en la Educación en Uruguay}

La Ley de Presupuesto Nacional quinquenal ${ }^{6}$, que se aprobó en 2015, establece la intención de realizar 439 obras nuevas (ya sean ampliaciones o construcciones totalmente nuevas) para toda la Administración Nacional de Educación Pública (ANEP) ${ }^{7}$ para el período 2016-2020. De estas 439 obras: 165 (38\%) son vía Contratos de PPP mientras que las restantes 274 (62\%) son con fondos propios de la ANEP, es decir, se van a realizar mediante el mecanismo tradicional de licitaciones públicas. Dentro de las obras de la ANEP se incluyen tanto edificios nuevos como ampliaciones y reparaciones en los centros educativos existentes, mientras que los de PPP son siempre edificios nuevos.

En el caso de las obras por Contratos de PPP, se elaboraron cuatro Proyectos de Infraestructura Educativa y para todos la ANEP ha optado por la modalidad DBFO (por la sigla en inglés), que incluye: el diseño, la construcción, el financiamiento y el mantenimiento de las diferentes obras.

A marzo de 2019 están los cuatro Proyectos de Infraestructura Educativa en marcha, aunque se encuentran en diferentes etapas y con distinto grado de avance. A modo de resumen, en el cuadro 1 se presentan los diferentes Proyectos de Infraestructura Educativa: 
Cuadro 1 - Descripción general de los Proyectos de PPP de la educación

\begin{tabular}{|c|c|c|c|}
\hline Proyecto & Empresas presentadas & Adjudicación & $\begin{array}{l}\text { Finalización } \\
\text { de obras }\end{array}$ \\
\hline $\begin{array}{l}44 \text { Jardines de Infantes } \\
15 \text { Centros CAIF }\end{array}$ & $\begin{array}{l}\text { Se presentaron } 3 \text { grupos de em- } \\
\text { presas: } \\
\text { Ebital SA, Aldesa Construcciones } \\
\text { SA, Nirazeld SA; } \\
\text { Berkes SA, Saceem, Stiler SA; } \\
\text { Basirey, Nelit, Conami }\end{array}$ & $\begin{array}{l}\text { Basirey, Nelit, } \\
\text { Conami }\end{array}$ & $\begin{array}{l}\text { Setiembre de } \\
2020\end{array}$ \\
\hline $\begin{array}{l}23 \text { Escuelas; } \\
9 \text { Polos Tecnológicos } \\
10 \text { Polideportivos }\end{array}$ & $\begin{array}{l}\text { Se presentaron } 3 \text { grupos de em- } \\
\text { presas: } \\
\text { Ebital SA, Aldesa Construcciones } \\
\text { SA, Nirazeld SA; } \\
\text { Berkes SA, Saceem, Stiler SA; } \\
\text { BTD Capital 12, Tecnove, Conami, } \\
\text { Basirey. }\end{array}$ & $\begin{array}{l}\text { Berkes, Sa- } \\
\text { ceem y Stiler. }\end{array}$ & Julio de 2020 \\
\hline $\begin{array}{l}15 \text { escuelas } \\
27 \text { CAIF }\end{array}$ & $\begin{array}{l}\text { Se presentaron } 3 \text { grupos de em- } \\
\text { presas: } \\
\text { Ebital SA, Aldesa Construcciones } \\
\text { SA, Nirazeld SA; } \\
\text { Berkes SA, Saceem, Stiler SA; } \\
\text { BTD Capital 12, Tecnove, Conami, } \\
\text { Basirey. }\end{array}$ & $\begin{array}{l}\text { A marzo de } \\
2019, \text { no ha } \\
\text { sido adjudi- } \\
\text { cada. }\end{array}$ & Fines de 2020 \\
\hline $\begin{array}{l}42 \text { liceos } \\
16 \text { UTU }\end{array}$ & $\begin{array}{l}\text { Se presentaron } 2 \text { grupos de em- } \\
\text { presas: } \\
\text { Ebital SA, Aldesa Construcciones } \\
\text { SA y Nirazeld SA; } \\
\text { Berkes SA, Saceem, Stiler SA y } \\
\text { Somediel SA. }\end{array}$ & $\begin{array}{l}\text { A marzo de } \\
2019, \text { no ha } \\
\text { sido adjudi- } \\
\text { cada. }\end{array}$ & En 2021 \\
\hline
\end{tabular}

Fuente: elaboración propia en base a información del Ministerio de Economía y Finanzas y ANEP.

Los cuatro pliegos significan: 42 edificios para la primera infancia -plan CAIF ${ }^{8}, 44$ para el preescolar -de 3 a 5 años-, 38 escuelas de primaria -de 6 a 12 años-, 42 liceos de educación media común, 25 UTU $^{9}$ y 10 plideportivos para educación media.

A su vez, hay dos grupos de empresas que se han presentado a los cuatro Proyectos de Infraestructura Educativa. Si bien el tercer y cuarto llamado no ha sido adjudicado, ambos podrían adjudicarse al mismo consorcio de empresas. Esto se debe a que los Contratos de PPP requieren un tamaño mínimo a efectos de justificar el costo de la contratación y facilitar las economías de escala necesarias para aumentar la eficiencia del funcionamiento y del 
mantenimiento. Esto genera proyectos de valores elevados donde solamente un pequeño número de operadores pueden ofrecer todos los productos y servicios solicitados. Así, la magnitud de los proyectos reduce el grado de competencia, ya que son pocas las empresas que cuentan con los recursos financieros y de infraestructura para presentar ofertas.

LOS costos

Como ya se mencionó, en los contratos de PPP para la Educación le corresponde al privado el diseño, la construcción, el financiamiento y el mantenimiento de las diferentes obras. Respecto al monto mensual que el Estado le transfiere al privado, a continuación, se presenta en el cuadro 2, un resumen con el Pago Por Disponibilidad máximo a pagar por mes, una vez que estén operativos los cuatro Proyecto de Infraestructura Educativa -y asumiendo que no hay renegociaciones de los contratos-.

Cuadro 2 - Erogaciones anuales por los 4 Proyectos de Infraestructura Educativa; expresadas en Unidades Indexadas (UI), pesos uruguayos (\$) y en dólares (USD)

\begin{tabular}{|l|l|l|}
\hline Total anual & $\begin{array}{l}\text { Pago Por Disponibilidad } \\
\text { (sin Impuesto al Valor } \\
\text { Agregado) }\end{array}$ & $\begin{array}{l}\text { Pago Por Disponibilidad } \\
\text { (con Impuesto al Valor } \\
\text { Agregado) }\end{array}$ \\
\hline En UI & UI 555.724.680 & UI 677.984.110 \\
\hline En \$ & $\$ 2.291 .364 .001$ & $\$ 2.795 .464 .081$ \\
\hline En USD & USD 67.234 .859 & USD 82.026 .528 \\
\hline
\end{tabular}

*Valor de la Unidad Indexada al 22-03-2019: \$4,1232. Valor del dólar (venta) al 22-03-2019: \$34,08. Tomado del Instituto Nacional de Estadísticas.

Fuente: elaboración propia en base al análisis de los estudios de factibilidad

Si tomamos el PBI del Banco Central del Uruguay para 2017 y las proyecciones de crecimiento para 2018 y 2019, el Pago Por Disponibilidad total anual representaría el 0,11\% del PBI de 2019, el 3,03\% del Gasto en Educación y el 4,26\% del presupuesto de la ANEP.

En relación a la moneda en el cual se realiza el pago, se establece que

Los Oferentes tienen la opción de solicitar que una porción de los PPD se denomine en dólares. Esta porción solicitada en dólares no podrá ser superior al 50\% y deberá ser indicada en el modelo resumen de la propuesta económica (Pliego de Condiciones Administrativas; 2018:14).

Originalmente, la totalidad de los PPD se iba a establecer en Unidades Indexadas. No obstante, luego del lobby empresarial, se modificó y se permitió el pago en dólares. Este dato no es menor, ya que da cuenta del poder del sector privado a la hora de negociar o renegociar este tipo de contratos con el Estado.

Por otro lado, una vez iniciada la etapa de operación de la obra, el privado deberá proporcionar los siguientes servicios obligatorios: mantenimiento rutinario y planeado; 
mantenimiento, reparaciones y reemplazo de daños; mantenimiento y reposición del equipamiento que corresponda; limpieza; mantenimiento del terreno; mantenimiento de instalaciones, edificios y activos y gestión del contrato e informes. Además, se establece que el privado

Podrá brindar servicios complementarios en los Centros una vez que estén en servicio. Para ello, deberá acordar las condiciones con la Administración Pública contratante y obtener su expresa autorización previa. Entre otros, podrá prestar servicio de cantina en tanto cumpla con lo exigido por las normas aplicables. (Proyecto de Contrato; 2018:6).

Es decir, desde los contratos ya se abre la posibilidad a que la empresa privada provea otros servicios en el centro educativo, abriendo la posibilidad para un mayor avance privatizador en la Educación. Por su parte, la ANEP proveerá los servicios educativos.

\section{Análisis de los Contratos de PPP}

Los pliegos de la licitación y el contrato que se firma entre la ANEP y la empresa privada establecen cuáles van a ser las reglas del juego. Se establece cuál va a ser la modalidad de construcción, cuál es el mobiliario con el que se debe equipar a los centros educativos, así como también la periodicidad con la cual se debe reponer. Además, menciona la frecuencia con la que se deben hacer tareas de limpieza y mantenimiento.

Dentro de este conjunto de pautas, se establecen los "días y horas de trabajo". A modo de ejemplo, el Pliego de Condiciones Técnicas para el caso de las instituciones educación media establece que: "Ios días de trabajo serán de lunes a viernes, desde el primer día hábil de febrero al 22 de diciembre de cada año, excepto períodos vacacionales y días feriados oficiales en Uruguay. En días de trabajo, las Horas de Trabajo podrán ir de las 7:00 a 23:45 horas." (2018:16)

También aparece el concepto de "Uso Mixto" que significa que la ANEP podrá hacer uso del Centro y el privado podrá realizar tareas de mantenimiento bajo ciertas condiciones. También se establece en el Pliego de Condiciones Técnicas que la ANEP

Tendrá derecho a establecer periodos de Uso Mixto dentro de los siguientes: en días de trabajo hasta las 22:00 horas (para Centros en que ese horario no sea de trabajo) y sábados de 8:00 a 18:00 horas, en hasta 45 ocasiones por año lectivo, para cada Centro educativo del objeto del contrato. Para ello, [...] deberá notificar con al menos 5 días de anticipación, la fecha y horario en que hará uso de este derecho. (2018:17).

Por lo tanto, por fuera de los días y plazos establecidos en los contratos, el privado dispone del centro educativo para actividades propias.

\section{Críticas}

Existe evidencia empírica internacional que muestra que este tipo de herramientas suelen ser más caras y problemáticas en relación a la inversión pública tradicional. Esta 
literatura, no solo proviene de autores o instituciones críticas de la privatización, sino incluso de los mismos organismos que promueven este tipo de herramientas como el FMI o la Unión Europea. En primer lugar, corresponde destacar el trabajo de Hall (2015), que muestra la experiencia de varios países donde hubo diversos fracasos de las PPP y el Estados termina asumiendo la administración de la infraestructura social. El resultado fue una obra y/o servicio más caro, cuyo costo recae sobre los usuarios, ya sea porque deben pagar más, o por la mala calidad del servicio. El autor también señala fracasos en los Contratos de PPP en la Educación, donde hubo diversos problemas: el servicio público educativo es desvirtuado por lógicas empresariales; los servicios educativos son de mala calidad e incluso casos donde nunca se terminaron las construcciones.

También se destaca el informe de la Internacional de la Educación (2009) que surge de un grupo de estudio a nivel mundial. Este informe da cuenta de los diversos tipos de asociaciones público privadas que existen para la educación y destaca los diversos problemas que surgen de estas modalidades.

Por otro lado, los organismos internacionales que impulsan y promueven este tipo de herramientas, también tienen informes técnicos que dan cuenta de los problemas del uso de los Contratos de PPP. En particular, aquí se destacan dos trabajos: por un lado, Informe del Tribunal de Cuentas de la UE (2018), donde muestra el resultado de una auditoría a varios proyectos de PPP en diferentes países europeos, mostrando que la mayoría no finalizaron a tiempo ni dentro del presupuesto previsto. El otro documento es del FMI (2004), donde se advierte sobre los problemas contables que trae aparejada las PPP para las finanzas públicas, ya que oculta buena parte del gasto fiscal futuro, lo que a su vez esto trae aparejados problemas de transparencia y de endeudamiento futuro. A continuación, resaltamos algunas de las críticas más importantes tomando los trabajos antes citados.

\section{Hay sobre costos y sobre plazos}

Según surge del Informe del Tribunal de Cuentas de la UE (2018), la mayoría de los proyectos fiscalizados no se finalizaron a tiempo ni dentro del presupuesto. Quienes defienden esta herramienta afirman que estos proyectos aumentan la eficiencia y logran terminar la construcción en el tiempo estipulado y dentro del presupuesto. Se asume que la empresa privada tiene un fuerte incentivo para finalizar las obras de construcción según lo previsto en su contrato, a fin empezar a recibir los Pagos Por Disponibilidad y evitar los aumentos de costes por los que suele asumir el riesgo. Sin embargo, el Tribunal constató que esto a menudo no se materializaron, ya que la infraestructura no se completó dentro del plazo y coste previstos. En siete de los nueve proyectos finalizados y analizados en el informe, equivalentes a un coste de proyectos de 7.800 millones de euros, los retrasos oscilaban entre 2 y 52 meses, y los aumentos de costes se acercaron a 1.500 millones de euros. 
En esta misma línea también aparecen dos trabajos de la Internacional de la Educación (2007; 2009), que muestran la experiencia en la construcción de 39 escuelas en Canadá a finales de la década del ' 90 . El primer contrato de arrendamiento se oficializó en 1998 y un año después, el nuevo gobierno canceló el proyecto porque se hizo evidente que las escuelas costaban más de lo que había sido previsto inicialmente, además de costar 32 millones de dólares más que si se hubiera financiado con recursos públicos.

\section{No necesariamente hay un mejor servicio}

Por otro lado, los defensores de este tipo de contratos suelen afirmar que la mayoría de estos proyectos mantienen un buen nivel de servicio y mantenimiento. Se afirma que los contratos de PPP garantizan mejores niveles de mantenimiento y servicio con respecto a los proyectos tradicionales. Detrás de esta afirmación, está la idea de que el socio del sector privado a cargo de la construcción también es responsable de la explotación y el mantenimiento de la infraestructura durante todo el lapso que dure el contrato, lo que supone más tiempo que el período habitual de garantía en virtud de las normas sobre contratación tradicional.

Para ello será necesario que el socio privado realice la planificación teniendo en cuenta los costes de explotación y mantenimiento a largo plazo que deberá asumir y la provisión de los niveles de servicio a los que se ha comprometido en el contrato de PPP. Por tanto, tendrá que prestar una atención especial a la calidad de la construcción. No obstante, la experiencia muestra que esto no se cumple necesariamente. En particular para la educación, la evidencia muestra que en los países que ya existía un servicio aceptable y extendido de educación pública, este tipo de modalidad suele deteriorar el servicio educativo existente, (IE, 2009).

\section{Hay una mala atribución y distribución de los riesgos}

El Tribunal de Cuentas de la UE (2018) encontró que las demoras, los aumentos de costes y la infrautilización, fueron en parte atribuibles a análisis inadecuados. En la mayoría de los proyectos fiscalizados, la opción de las PPP se eligió sin un análisis comparativo previo que permitiera demostrar que se trataba de la mejor optimización de recursos.

Por otro lado, la transferencia de riesgo es un factor fundamental a la hora de comparar la opción de una PPP frente a la licitación tradicional. La idea de fondo es que el riesgo lo debe asumir el socio que esté en mejores condiciones de gestionarlos. Sin embargo, la asignación del riesgo fue con frecuencia inadecuada, lo que dio lugar a una exposición al riesgo excesiva para el socio privado. El problema radica en que es muy difícil detectar y asignar riesgos de proyectos correctamente. 
Para el caso de la educación siempre es el gobierno el que carga con la responsabilidad final a la hora de proporcionar los servicios públicos, por lo que la asunción de la "distribución del riesgo" es discutible (IE; 2009).

\section{Puede haber falta de transparencia en los Contratos de PPP}

La posibilidad de registrar los Contratos de PPP como partidas fuera de balance financiero de la Administración Pública, hace de esta herramienta una opción tentadora para los gobiernos que quieren invertir en infraestructura social y no afectar el déficit fiscal. Según la UE, esta consideración estadística revierte mucha importancia para las autoridades públicas a la hora de decidir si recurren o no a este tipo de contratos.

El hecho de mantener los Contrato de PPP fuera del balance público significa trasladar gran parte de los riesgos y beneficios al socio privado. En ese sentido, existe un riesgo inherente de que los contratos se vean influenciados por el tratamiento estadístico preferencial en lugar de por el principio de que debería asumir los riesgos la parte que esté en mejor posición para gestionarlos y maximizar la relación calidad-precio.

Es así que, mantener los Contratos de PPP fuera de los balances públicos reduce el nivel de información transparente facilitada al público en general sobre los compromisos asumidos a largo plazo con esta herramienta y por lo tanto, sobre su incidencia en los niveles de deuda y déficit de los Estados.

\section{Se desdibuja el espacio público y se precariza el trabajo}

El Estado ya no es dueño del espacio donde funciona el centro educativo, sino que lo arrienda a una empresa privada durante el tiempo que estipule el contrato. Esto es un cambio no menor, ya que donde antes funcionaba un espacio público, ahora queda librado a la voluntad de una empresa privada, que dispone de su uso en una parte importante del tiempo. Es no menor tener presente que la vida de los centros educativos trasciende las actividades previstas por la ANEP. De hecho, muchas actividades se desarrollan fuera del horario de clases: emergentes educativos, actividades de integración, salidas didácticas, convocatoria a padres, actividades de formación, eventos solidarios, muestras artísticas, etc. Así, se restringe toda iniciativa extracurricular de la comunidad educativa.

Con respecto a la situación laboral, en el caso de los docentes no habrá cambios ya que los Contratos de PPP no afectan a los servicios educativos. Sin embargo, los trabajadores que se encarguen de las tareas de mantenimiento y limpieza serán trabajadores del sector privados. No obstante, esto no es algo nuevo. En muchos centros centros educativos, ya se da una subcontratación de servicios de apoyo no educativos que según la definición manejada por la Internacional de la Educación (2009), también es una forma de PPP. 


\section{La ley de mecenazgo}

Como se destaca en la introducción, históricamente las instituciones de enseñanza privada en Uruguay cuentan con exoneraciones impositivas totales. El artículo 69 de la constitución vigente establece que "las instituciones de enseñanza privada y las culturales de la misma naturaleza estarán exoneradas de impuestos nacionales y municipales, como subvención por sus servicios".

A partir de la Ley 18.083 de 2007 (Ley de la Reforma Tributaria) nace otro mecanismo de exoneración impositiva destinado a instituciones de enseñanza privada. Los artículos 78 y 79 modifican una vieja ley conocida como "Ley de Mecenazgo", permitiendo que empresas privadas hagan donaciones a instituciones de educación -tanto públicas como privadas- a cambio de exoneraciones fiscales. Según los artículos referidos, sobre el monto donado, el Estado exonera de impuestos a las empresas donantes en un 75\% por concepto del Impuesto de la Renta de la Actividad Económica (IRAE) e Impuesto al Patrimonio. El 25\% restante, las empresas pueden calificarlo como gasto de la empresa aumentando la exoneración impositiva. En suma, del total donado, el Estado le devuelve a las empresas el $83 \%$ del monto.

En la Ley de Rendición de Cuentas de 2013 -Ley N¹9.275-, el artículo 358 modificó la lista de organizaciones pasibles de recibir donaciones. Allí se incorporó la posibilidad de que las empresas puedan donar dinero a instituciones privadas de la primera infancia, la educación primaria, secundaria, técnico-profesional y que atiendan a poblaciones más carenciadas. Antes de esta modificación sólo podían donar a instituciones del Estado y las donaciones a instituciones privadas bajo este régimen sólo podían ser de útiles, vestimenta, construcciones y reparaciones en este tipo de establecimientos.

Además, se modificó el monto máximo que las empresas podrían donar para descontar impuestos. Según la normativa, las empresas no pueden donar más que el $5 \%$ de la renta fiscal neta del ejercicio anterior, pero se agregó la excepción que en caso de que el destino sean instituciones nombradas en el anterior párrafo, el máximo a donar pasa a ser el $10 \%$ de la renta fiscal neta, siempre y cuando las donaciones sean mayores o iguales a las del año anterior.

Así, estas modificaciones permitieron consolidar e incrementar esta nueva oferta educativa y fortalecer en varios ámbitos de discusión (y en los hechos, en el mismo Estado), la idea de que la solución a la "crisis educativa" en los contextos más vulnerables, vendría de la mano de instituciones educativas privadas gratuitas. Las cuales, a su vez, deben tener un fuerte vínculo con el mundo empresarial (con el argumento de que prepararía mejor a las y los jóvenes para el mundo del trabajo). Esto generó un cambio en el destino de las donaciones. Mientras que, en 2010, casi la totalidad de lo donado -98,43\%- fue a la Administración Nacional de Educación Pública (ANEP), en 2017 el grueso de las donaciones -93,96\%- fue para centros educativos de gestión privada. 
Esta modalidad genera una pérdida del control sobre la oferta educativa ya que el Estado es el principal financista vía exoneración de impuestos, pero no tiene ningún tipo de incidencia en el nacimiento y el funcionamiento de estos centros educativos. Esta situación se hace más grave teniendo en cuenta que hay entidades públicas de derecho privado como la Administradora de Fondos de Ahorro Previsional estatal República AFAP, que realiza donaciones a instituciones de educación media de gestión privada, como es la Fundación Impulso.

A su vez, con esta modificación de la Ley de Mecenazgo, se abrió la posibilidad de crear una oferta educativa no registrada en Uruguay hasta ese momento, similares a las conocidas en la literatura como escuelas tipo "charters". En general las escuelas "charter", o las escuelas "concertadas", son centros educativos públicos administrados por empresas privadas que reciben financiamiento por parte del Estado. El mismo está atado a la cantidad de alumnos, así como a su rendimiento académico. Su atractivo está en tener independencia de la administración pública educativa central o de los distritos escolares, lo que les permite: generar formas innovadoras en cuanto a metodologías pedagógicas; implementar esquemas de incentivos a su plantilla de profesores; contratar y despedir profesores con mayor facilidad, lo que incrementa la inestabilidad y la precariedad laboral.

Para el caso de Uruguay, no es posible hablar de escuelas "charter" tal cual se hace en el resto del mundo. Esto se debe a que los centros educativos que más se acercan a esta dinámica son privados. Igualmente pueden generarse comparaciones dado que estos centros privados reciben recursos estatales de manera indirecta a través de la renuncia tributaria generada por las donaciones que los financian.

Las empresas donantes pertenecen a los más diversos rubros, destacándose las donaciones que realizan los Bancos. A modo de ejemplo, tomando los datos publicados en la Rendiciones de Cuentas del 2017 se puede ver que las dos empresas que más han donado a instituciones educativas han sido, en primer lugar, el Banco Santander que en 2017 donó poco más que $\$ 42$ millones de pesos a instituciones privadas y a Facultades y Fundaciones de la Universidad de la República. En segundo lugar, se ubica el Banco Itaú ${ }^{10}$ que en 2017 donó poco más de \$14 millones de pesos.

Además, según los datos de las Rendiciones de Cuentas, podemos observar que la mayoría de las donaciones estaban dirigidas a instituciones ya existentes, con un fuerte sesgo hacia el nivel terciario (Universidades Privadas, Fundaciones de Facultades de la Universidad de la República, Facultades de la Universidad de la República y sus oficinas centrales). No obstante, luego de las modificaciones a esta Ley en 2013, se crearon 3 escuelas de primaria, 4 de secundaria y 1 de técnica que empezaron a acaparar las donaciones.

Por otro lado, las donaciones hacia instituciones educativas de gestión privada generan una mayor desigualdad respecto a los centros educativos públicos, ya que cuentan con mayor disponibilidad de recursos. En este sentido, si tomamos como ejemplo al Liceo Jubilar -un centro educativo de gestión privada cuya fuente de financiamiento está asociada 
a las donaciones-, y analizamos el gasto por alumno, se puede ver mejor este fenómeno (ver gráfico 1). En 2017, el gasto corriente anual por alumno (excluyendo las inversiones) en la secundaria pública fue de $\$ 65.877$, mientras que en el Liceo Jubilar fue de $\$ 181.939$.

Gráfico 1 - Comparación gasto por alumno en pesos corrientes entre el Consejo de Educación Secundaria de la ANEP y el liceo Jubilar.

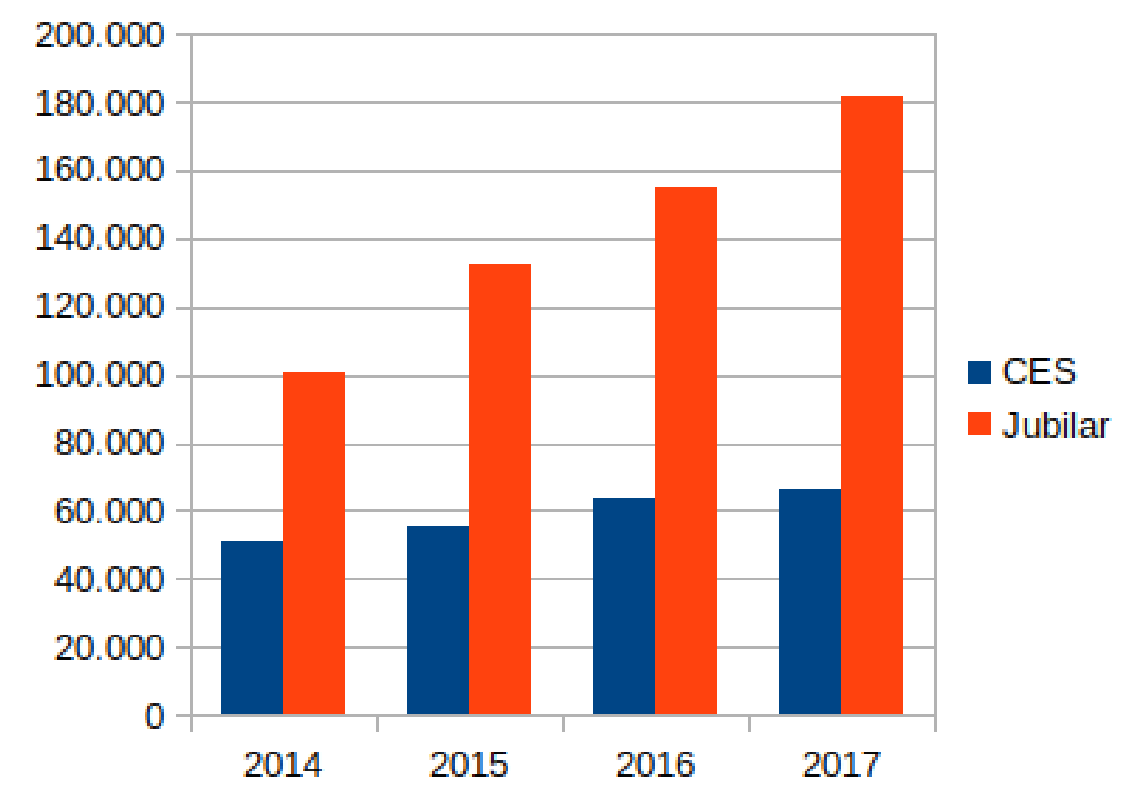

Fuente: Elaboración propia en base a datos de Contaduria General de la Nación, datos administrativos de ANEP, Rendición de cuentas 2014, 2015, 2016 y 2017.

Esta situación desigual, se viene manteniendo desde la creación de este centro educativo en 2013. Por cada peso que disponía el Consejo de Educación Secundaria de la ANEP, el Liceo Jubilar contaba con $\$ 1,97$. Conforme pasan los años, esta diferencia se va haciendo cada vez más grande y en 2017, por cada peso que tiene el sistema público, el Liceo Jubilar cuenta con $\$ 2,76$.

A su vez, es importante resaltar que el Estado termina haciendo un gran esfuerzo económico en este tipo de instituciones. Una vez más, tomando como ejemplo al Liceo Jubilar, vemos que las donaciones recibidas por esta institución en 2017 ascienden a $\$ 12.003 .922$. No obstante, solo $\$ 2.040 .667$ fue efectivamente aportado por las empresas y el resto fue asumido por el Estado vía exoneración impositiva. Por otro lado, resulta interesante comparar el gasto por estudiante de la educación media estatal, con el gasto tributario (es decir, lo que el Estado deliberadamente deja de recaudar de impuestos) por estudiante en función de las donaciones recibidas por el Liceo Jubilar. En este sentido, si miramos el gasto tributario por alumno, $\$ 49.816$ fue devuelto por el Estado a las empresas aportantes en el 2017. Al tomar al Gasto Tributario como una de las formas del gasto público estatal, podemos ver que el Estado gasta en este tipo de educación privada más recursos por alumno que los que les destina a los estudiantes de secundaria pública. 
El resultado fuera de la tendencia del último año puede ser debido a que el Liceo Jubilar fue la única institución de educación privada de enseñanza secundaria que recibió menos donaciones en 2017 en comparación al año anterior (tomado en pesos corrientes), lo cual dejó menor espacio para generar Gasto Tributario. Esto tiene que ver con la creación de otras entidades de educación media que disputan las donaciones con el Liceo Jubilar (Bachillerato Tecnológico Ánima, Liceo Providencia, etc.), y el aumento de las donaciones a otras entidades como la Fundación Impulso que pasó de acaparar el $50 \%$ de las donaciones a instituciones de la enseñanza primaria, secundaria y técnica en 2016, a recibir el 55\% en 2017.

Lo mismo puede verse para otra de las instituciones insignia de esta modalidad de liceos privados gratuitos, la Fundación Impulso. Desde 2015 (primer año con que se cuenta información del balance en la Rendición de Cuentas), por cada peso por alumno que gasta el CES, el Impulso gastó \$2,63 en 2015; \$2,2 en 2016; y \$ 2 en 2017.

El apoyo Estatal a este tipo de instituciones es cuestionable por otro lado, debido a la lógica de selección del estudiantado que tienen estas instituciones. Como señala De Melo (2013) gran parte del proceso de selección refiere al interés, apoyo y compromiso que tienen las familias de los estudiantes para con el proceso educativo. Esta característica es muy importante para analizar los fenómenos de la deserción y el rendimiento curricular. En todos los subsistemas educativos, es razonable que les vaya mejor a los estudiantes que ven un propósito en asistir a las instituciones educativas, así como los que tienen una familia que los apoya en esa decisión. Por lo tanto, a pesar de que estas instituciones se concentren en contextos de vulnerabilidad socioeconómica (donde existen mayores tasas de deserción educativa), sólo captan los estudiantes que tendrían menos posibilidades de desertar en cualquier sistema educativo.

Por otro lado, durante el 2016 se aprobó en la Rendición de Cuentas una propuesta de modificación a la Ley 18.083, reduciendo las posibilidades de exoneraciones de impuestos al 50\% en las donaciones especiales a las Universidades Privadas. Esto generó un descenso en el último año en las donaciones recibidas por estas instituciones. 
Gráfico 2 - Donaciones recibidas por Universidades Privadas en pesos corrientes, y proporción de recepción de donaciones de las Universidades Privadas en relación al total de donaciones destinadas a la educación privada.

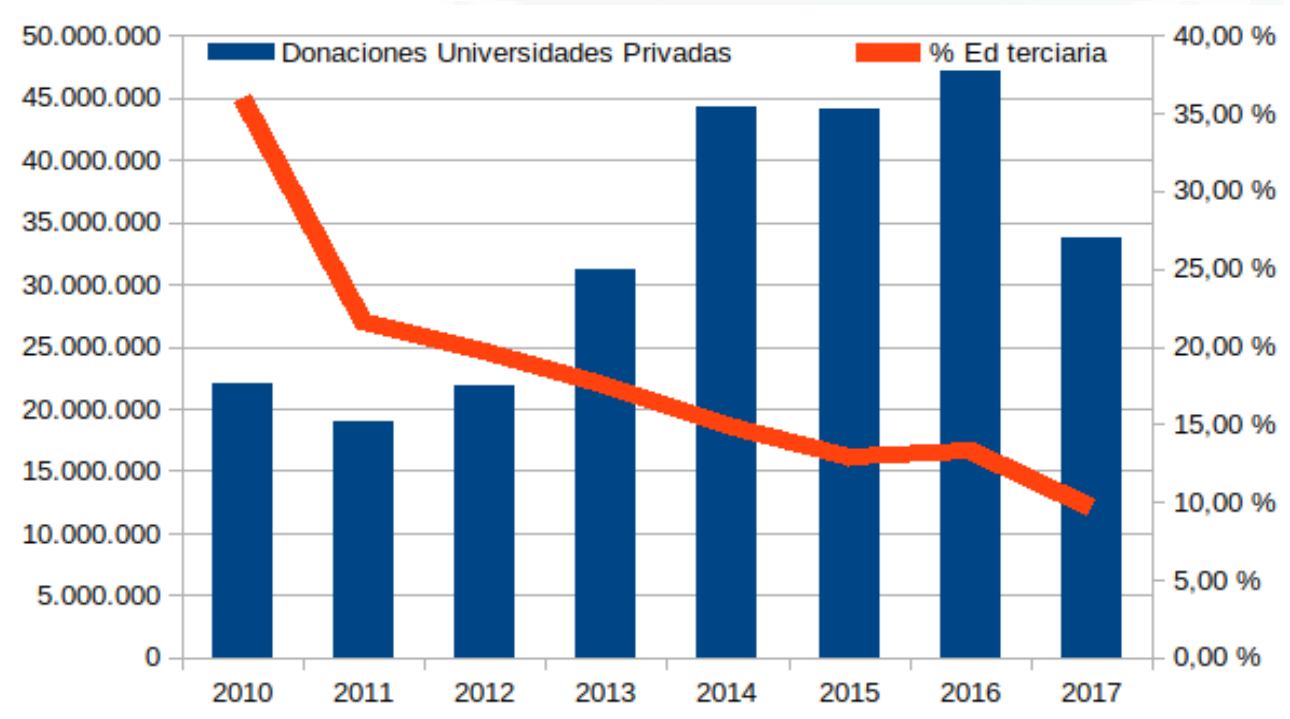

Fuente: Elaboración propia en base a datos de Rendiciones de Cuentas Ejercicios 2010 - 2017

En el gráfico 2, se pueden ver dos procesos. En primer lugar, cómo en términos brutos, las Universidades Privadas recibieron menor cantidad de recursos en el año 2017. Este fenómeno marca un quiebre con respecto a lo que podía ser una tendencia de crecimiento desde el año 2011. En segundo lugar, en todo el período hay una tendencia clara donde la proporción de donaciones que están destinadas a las Universidades Privadas en relación al total del sistema educativo cae. Esto además de explicarse por el fenómeno mencionado anteriormente con respecto al último año, tiene que ver con la emergencia de nuevos centros educativos pasibles de recibir donaciones como los liceos privados gratuitos.

Como consecuencia de la modificación en la normativa, todas las Universidades privadas recibieron menos donaciones en el año 2017 con relación al 2016:

Cuadro 3 - Variación de la cantidad de donaciones recibidas por las Universidades Privadas entre 2016 y 2017

\begin{tabular}{|l|l|}
\hline Institución & Variación 2017 - 2016 \\
\hline CLAEH & $-37,46 \%$ \\
\hline UCUDAL & $-11,99 \%$ \\
\hline Universidad de la Empresa & $-21,10 \%$ \\
\hline Universidad de Montevideo & $-28,56 \%$ \\
\hline Universidad ORT & $-49,85 \%$ \\
\hline
\end{tabular}

Fuente: elaboración propia en base a datos de Rendición de Cuentas Ejercicios 2010 - 2017 
Desde la modificación de la ley de Mecenazgo en 2013, el 2017 fue el primer año de descenso del monto en las donaciones a dichas universidades privadas. Hasta ese entonces todas a excepción del año 2015 para la Universidad de Montevideo y la Universidad ORT habían presentado un contínuo crecimiento año a año.

El comportamiento del monto de donaciones a las Universidades Privadas demuestra (a pesar de que sólo se cuenta con un año para la comparación) de que, en las donaciones prima, o por lo menos es determinante, la posibilidad de los empresarios para reducir su carga impositiva a la caridad o altruismo de las empresas. Este comportamiento deja en evidencia que es inviable desde el punto de vista financiero, pensar este tipo de herramientas como una solución universal o masiva, ya que dependen de incentivos privados y muy asociados al ciclo económico.

Además, este tipo de políticas educativas deja muy expuesta la posibilidad de que las empresas puedan hacer o influir en las políticas educativas según sus intereses. Un ejemplo de ello puede verse en las donaciones a la Universidad de la República, donde se refleja un interés específico de las empresas donantes en las Facultades que reciben la donación. Por ejemplo, a la Facultad de Agronomía le donan empresas vinculadas al agronegocio como Barraca Erro o Lebú SRL; a la Facultad de Ciencias le dona el Laboratorio Celcius; a Facultad de Química le dona la empresa Bayer S.A. y a Facultad de Ciencias Económicas el Banco Santander. Este tipo de donaciones ayuda a generar un vínculo más fuerte entre determinado tipo de insumo o práctica con el futuro profesional, lo que implicaría una relación más fuerte y una dependencia tecnológica una vez egresado. A la vez, condiciona la puesta en práctica de cursos o proyectos al aporte privado de insumos o recursos.

\section{Reflexiones finales}

El documento analiza dos tipos de herramientas novedosas en el Uruguay que logran dar un paso más en la privatización exógena del modelo educativo uruguayo. Estas herramientas se corresponden con una nueva oleada internacional y regional sobre la educación pública y por la disputa de los recursos públicos.

Una de las herramientas son las PPP, muy utilizadas para privatizar los servicios públicos como la educación y la salud en lo que autores como Fine y Hall (2012), llaman la nueva oleada privatizadora. En nuestro país tuvieron su marco legal en el año 2011, pero es a partir del presupuesto nacional del año 2015 que comenzó a aplicarse a gran escala. En particular, en la educación en este quinquenio se implementaron 4 pliegos que afectan a la primera infancia, la educación inicial, primaria y la media. Ya están en marcha la construcción de 201 centros de estudio bajo esta modalidad.

Esto tiene un gran impacto en lo que hace a la privatización de buena parte del sistema educativo uruguayo, y es una gran cantidad de recursos que se desvían del sector público al privado por al menos 20 años. 
Esta modalidad constructiva no es solo la privatización de la construcción, sino de todos los servicios no educativos que hacen a la institución. Las empresas privadas serán dueñas de estos centros educativos por 20 años una vez finalizada la construcción, y tiene la opción de extender el contrato hasta 35 años. Más allá de las críticas económicas que se le puedan hacer a este tipo de herramientas, son en los hechos, una privatización de un espacio público, ya que ahora ese espacio es regido por lógicas del mercado y tienen un dueño privado.

Existe evidencia empírica que muestra que este tipo de herramientas privatizadoras suelen traer más problemas y terminan siendo más caras que lo previsto y que las construcciones de tipo tradicional. Esto es, debido a que suelen subestimarse los riesgos y los privados se rigen por las lógicas del mercado, no por un fin social. También existe evidencia específica de este tipo de instrumentos aplicados a la educación que muestra muy malos resultados en lo que hace al sistema educativo.

A futuro es dable esperar más problemas, es lo que muestra la evidencia empírica mencionada. Las empresas que ganaron las licitaciones son un holding muy poderoso que suelen presionar con éxito a los gobiernos con el fin de obtener ganancias extraordinarias. Los sistemas educativos precisan cambios y adaptaciones para adaptarse a las realidades locales, los contratos suelen ser de muy largo plazo, y en las renegociaciones de los mismos suelen ganar grandes ventajas los privados, atando al sistema educativo a las necesidades privadas. A su vez, también es dable esperar que estas empresas quiebren o tomen decisiones empresariales en direcciones opuestas al bienestar social, haciendo encarecer y perjudicar el sistema educativo.

Otra de las herramientas analizadas es modificación a la Ley de Mecenazgo, donde se generaron incentivos fiscales para la donación de empresas a instituciones educativas privadas. Si bien en lo que hace al impacto en la matrícula y en la captación de recursos no parece ser significativo en la actualidad, en el plano simbólico ha generado un gran impacto. Variados actores políticos, académicos y generadores de opinión ponen a este modelo como la luz para acabar con los "problemas" de la educación (Bordoli et al 2017).

De acuerdo con la investigación impulsada por la Internacional de la Educación "Privatización educativa en Uruguay: políticas, actores y posiciones", es destacable la importancia que tiene este mecanismo de transferencia fiscal del Estado a la educación privada "a diferencia de la legislación de otros países de la región, no existe en Uruguay sistemas de subvención directa a las instituciones privadas. Sin embargo, el régimen de exoneraciones resulta más abarcativo que los existentes en otros países con sistema de subvención y constituye, por tanto, una fuente de ingresos indirectos de vital relevancia para las instituciones privadas en el país." (Bordoli et al., 2017:26)

El resultado de esta política fue la consolidación y el incremento de oferta educativa privada, principalmente en sectores que no existía como zonas de contexto socioeconómico vulnerable a través de centros educativos privados gratuitos. Esto se dio 
de la mano del cambio en la dirección de las donaciones por parte de las empresas hacia el sistema educativo. Las modificaciones realizadas a la Ley de Mecenazgo permitieron que estas se derivaran del sector público al privado.

Por otra parte, este nuevo direccionamiento de las donaciones permitió la aparición de centros educativos privados con lógicas operacionales nuevas. En este sentido, es que aparecen los liceos gratuitos de gestión privada, y lógicas de funcionamiento parecidas a las conocidas como escuelas "charter" o "concertadas".

Por último, merece la pena destacar que con este mecanismo de promoción de donaciones, el Estado voluntariamente resigna su papel de redistribuidor de recursos en la economía. En lugar de recaudar impuestos para destinarlos en servicios públicos capaces de alcanzar a todos sus habitantes, resigna ingresos fiscales para que los sectores capitalistas inviertan en el tipo de servicios que a ellos les interesa. Esto genera formaciones de estudiantes o futuros profesionales dependientes a cierto tipo de insumo o tecnología para la práctica de la actividad, o centros educativos que al tener mecanismos selectivos ayudan a estigmatizar aún más las personas dentro de su comunidad. A su vez, es una política que desde el punto de vista financiero es limitada pensando su expansión y puede tener problemas financieros en el corto y mediano plazo, ya que depende de donaciones y por tanto, del ciclo de la economía.

\section{Referencias}

AGUIAR, C. Uruguay: país de emigración. Montevideo: EBO, 1982

BORDOLI, E.; et al. Privatización educativa en Uruguay: políticas, actores y posiciones. Internacional de la educación, Montevideo. 2017. ISBN 978-92-95109-46-9

BRUM, S.; SILVA, K. Evolución de la educación secundaria en los últimos cincuenta años. Desafíos para la próxima década. Concurso para investigadores jóvenes “El Informe de Educación de la CIDE 50 años después", Montevideo: INEEd, 2013.

BUCHELI, M.; CABELLA, W. El perfil demográfico y socioeconómico de la población uruguaya según su ascendencia racial. Notas de Población, Santiago de Chile, n 91, 2010. ISBN 978-921-323446-3.

CASTILLO, J.; et al. El gasto en educación en Uruguay y su repercusión en las condiciones de trabajo de los docentes. Una mirada histórica y comparada. Revista Contrapunto, Montevideo, Volumen 7, 2015. ISSN: 2301-0282.

DE MELO, G. Educación: ¿encontramos el modelo? Blog Razones y Personas, Montevideo, 2013. http://www.razonesypersonas.com/2013/04/educacion-habemus-el-modelo_4.html

ESPONDA, F.; MOLINARI, J. Reglas de juego de la inversión pública. El caso de la revolución eólica uruguaya. Lecciones para las PPP. Trabajo presentado en $9^{\circ}$ Congreso Latinoamericano de Ciencia Política, organizado por la Asociación Latinoamericana de Ciencia Política, Montevideo, 2017. 
FERNÁNDEZ ENGUITA, M. Trabajo, escuela e ideología. Madrid: Akal, 1985.

FINE, B.; HALL D. El terreno del neoliberalismo: obstáculos y oportunidades para el desarrollo de Modelos alternativos en el suministro de servicios. En Alternativas a la privatización, Montevideo, 2012. ISBN: 978-84-9888-471-5

FMI. Fondo Monetario Internacionao. 2004. «Public Private Partnerships»

http://www.servicesforall.org/html/Privatization/IMF_Public_Private_Partnerships.pdf

HALL, D. ¿Por qué las asociaciones público-privados no funcionan? Las numerosas ventajas de la alternativa pública. Londres: Public Services International Research Unit, 2015.

INEEd. Informe sobre el estado de la educación en Uruguay 2015-2016. Montevideo: INEEd, 2017.

MARCONI, C.; LARA, C. Evolución de la educación inicial y primaria en los últimos 50 años. Desafíos para la próxima década. Concurso para investigadores jóvenes “El Informe de Educación de la CIDE 50 años después", Montevideo: INEEd, 2013.

MFE. Ministerio de Economía y Finanzas. Rendición de Cuentas 2017. Uruguay: MFE, 2017. https://www.mef.gub.uy/24681/1/mef/rendicion-de-cuentas-y-balance-de-ejecucionpresupuestal-2017.html

NAHUM, B. Estadísticas históricas del Uruguay 1900-1950. Montevideo: Universidad de la República, 2007. ISBN: 978-9974-0-0377-4

PNUD. Política, políticas y desarrollo humano. Montevideo: PNUD, 2008.

STOLOVICH, L. La cuestión salarial. Montevideo: CIEDUR, 1988.

TALVI, E. Propuesta de Talvi en la construcción de 136 liceos tipo charter. 2018. https:// www.talvi.uy/propuestas

TRIBUNAL DE CUENTAS EUROPEO. Asociaciones público-privadas en la UE: Deficiencias generalizadas y beneficios limitados. Informe especial No 9, 2018.

VERGER, A.; BONAL, X. La emergencia de las alianzas público-privadas en la agenda educativa global: Nuevos retos para la investigación educativa. Revista Profesorado, n 16, 2012. ISSN 1989-639X (edición electrónica)

VERGER, A.; BONAL, X. y ZANCAJO, A. What Are the Role and Impact of Public-Private Partnerships in Education? A Realist Evaluation of the Chilean Education Quasi-Market. Comparative Education Review, Chicago, n 60, 2016.

VERGER, A.; FONTDEVILA, C., y ZANCAJO, A. La economía política de la privatización educativa: políticas, tendencias y trayectorias desde una perspectiva comparada. Revista Colombiana de Educación, Bogotá, n 70, 2016.

Artigo recibido en: 25/04/2019 Aprobado en: 03/06/2019

\section{Contacto para correspondencia:}

Martín Jauge Caneiro.E-mail: martinjauge@gmail.com 
1 Se refiere al sistema por el cual el Estado garantiza a cada estudiante un monto de dinero o cápita por el cual puede optar por usar en cualquier establecimiento educativo de la oferta disponible para comprar educación.

2 Tomando los anuarios del Ministerio de Educación y Cultura, en lo que hace a la enseñanza formal obligatoria -de 4 años a la finalización de la educación media- en el 2016 la primaria privada era el $18 \%$ de la matrícula total, en media toda es del $12 \%$ y la universidad tiene tasas del $15 \%$ de matrícula privada.

3 Las siglas significan Asociación Uruguaya de Educación Católica.

$4 \quad$ Ejemplo de estas reglas pueden verse en todos los países, pero los más comunes: la Unión Europea fija un Déficit Fiscal máximo del 3\% del Pib (criterios de Maastricht); en América Latina hay reglas de contabilidad pública que limitan la inversión sumado a leyes que ponen topes de inversión pública.

$5 \quad$ En 1989 el Fondo Monetario Internacional obligó a los Estados a contabilizar la inversión pública de esta manera.

6

Ley No 19.355.

7 La ANEP es el organismo público que se encarga de brindar la oferta educativa pública y de la regulación del sistema educativo en los niveles: preescolar ( 3 a 5 años), primaria (6 a 12 años), media -regular y técnica- y de la formación docente para dichos niveles.

8 El plan CAIF -Centros de Atención integral a la primera Infancia y la familia-, data de 1988 y es la atención que brinda el Estado vía Organizaciones Civiles y Sociales a los y las niñas de 0 a 3 años. Este plan depende del Instituto del Niño y Adolescente del Uruguay INAU.

9 La Universidad del Trabajo del Uruguay, se encarga de enseñanza media (básica y superior) y de enseñanza terciaria.

10 Se contabilizan las donaciones realizadas por todo el grupo económico, por lo que también se incluyen empresas como la tarjeta de crédito OCA o Unión Capital AFAP. 